Revue

Revue de l'histoire des religions

de Ihistoire

des religions

3 | 2018

Varia

\title{
L'autre modernité du sujet. Foucault et la confession de la chair : les pratiques de subjectivation à l'âge des Réformes
}

The Other Modernity of the Subject. Foucault and the Confession of the Flesh:

Subjectivation Practices in the Reformation Period

\section{Arianna Sforzini}

\section{OpenEdition}

\section{Journals}

Édition électronique

URL : http://journals.openedition.org/rhr/8955

DOI : $10.4000 /$ rhr.8955

ISSN : 2105-2573

Éditeur

Armand Colin

\section{Édition imprimée}

Date de publication : 1 septembre 2018

Pagination : 485-505

ISBN : 978-2-200-93188-9

ISSN : 0035-1423

\section{Référence électronique}

Arianna Sforzini, «L'autre modernité du sujet. Foucault et la confession de la chair : les pratiques de

subjectivation à l'âge des Réformes », Revue de l'histoire des religions [En ligne], 3 | 2018, mis en ligne le 01 septembre 2020, consulté le 13 janvier 2021. URL : http://journals.openedition.org/rhr/8955 ; DOI : https://doi.org/10.4000/rhr.8955 


\section{L'autre modernité du sujet Foucault et la confession de la chair : les pratiques de subjectivation à l'âge des Réformes}

Les fragments du deuxième tome inédit de l'Histoire de la sexualité dans son premier projet (La chair et le corps) nous montrent que l'étude du christianisme du début de l'époque moderne est un moment essentiel pour la production foucaldienne. Au milieu des années soixante-dix, à travers l'étude d'un problème concernant l'histoire à la fois religieuse et sexuelle (la confession du péché de luxure entre Réforme et ContreRéforme), Foucault rencontre une dimension inédite du rapport à soi, à son corps et à sa parole de vérité. Il introduit, dans ses analyses, une problématique subjective qui, pour n'avoir jamais été absente de ses préoccupations, n'était toutefois pas pensée dans cette centralité de la relation à soi comme possibilité ouverte à la fois de conflit et de liberté.

\section{The Other Modernity of the Subject.}

Foucault and the Confession of the Flesh :

Subjectivation Practices in the Reformation Period

The remaining fragments of the second unpublished tome of the History of sexuality (Flesh and Body) show us that the reflection on early modern Christianity is a capital moment in Foucault's production. In the mid-1970s, studying both a religious and sexual problem (the confession of the sin of lust between the Reformation and the Counter-Reformation), Foucault encounters a new dimension of the self, constituted as a relationship to one's own body and truth-discourses. In his analysis, he introduces the issue of subjectivity, which was never absent from his intellectual works but was not yet considered within this central relationship to the self as an open possibility both of conflict and of freedom. 


\section{L'HISTOIRE INACHEVÉE DE LA SEXUALITÉ}

Les études foucaldiennes ont aussi leurs lieux communs. Parmi eux, l'idée que Michel Foucault, critique invétéré des dispositifs discursifs et politiques, chef de file de la postmodernité dans toute sa portée anti-transcendantaliste et anti-universelle, se serait « converti » à l'éthique au tournant des années quatre-vingt. Selon cette idée, il aurait précisément retrouvé comme axe directeur de sa réflexion, la dimension de la subjectivité que sa philosophie, depuis ses premiers travaux, s'était acharnée à réfuter et rendre inopérante. Dans le long processus de réélaboration du projet de l'Histoire de la sexualité, après une «immersion» dans l'histoire du (néo-)libéralisme qui questionna son public et irrita ses amis gauchistes, Foucault se serait découvert beaucoup moins blanchotien qu'il ne croyait l'être, plus sartrien peut-être, s'engageant dans une toute nouvelle entreprise de pensée de la liberté et du sujet « sculpteur » de sa propre existence.

Comme tout lieu commun, celui-là aussi a ses fondements de vérité ainsi que ses limites et ses éléments de naïveté. Je ne veux pas entrer ici dans un débat complexe qui dure depuis plusieurs années et qui a été longuement traité par des commentateurs avertis $^{1}$. Je souhaiterais tout simplement contribuer à dévoiler un passage encore en grande partie méconnu de la pensée foucaldienne à propos des recherches menées au milieu des années soixante-dix et qui devaient donner corps au projet du deuxième volume de l'Histoire de la sexualité dans sa première version ${ }^{2}$ : La chair et le $\operatorname{corps}^{3}$. Je proposerai une hypothèse de travail qui

1. Pour une reconstruction du parcours foucaldien et des difficultés liées à la « discontinuité » de ses analyses, voir en particulier Judith Revel, Foucault, une pensée du discontinu, Paris, Mille et une nuits, 2010. Voir aussi l' "Introduction » de Frédéric Gros à Michel Foucault, CEuvres, t. 1, Paris, Gallimard («Pléiade »), 2015, p. IX-XXXIII.

2. Le plan d'une histoire de la sexualité en six volumes était donné à lire sur la quatrième de couverture de la première édition de La volonté de savoir en 1976 : 1. La volonté de savoir; 2. La chair et le corps; 3. La croisade des enfants; 4. La femme, la mère et l'hystérique; 5. Les pervers; 6. Populations et races.

3. Dans la «Chronologie» de la vie intellectuelle de Foucault, Daniel Defert affirme que La chair et le corps se présentait comme « une généalogie de la concupiscence à travers la pratique de la confession dans le christianisme 
me paraît féconde pour comprendre non seulement ces analyses inédites, mais plus généralement la manière dont ces dernières influent sur le parcours de Foucault jusqu'à la fin de sa vie, jusqu'au «tournant éthique » qui caractérise ce qu'il définit luimême en 1984 comme son «trip gréco-latin » ${ }^{4}$. On peut résumer cette hypothèse ainsi : c'est à travers l'étude d'un problème bien spécifique concernant l'histoire à la fois religieuse et sexuelle moderne (la confession du péché de luxure entre le $\mathrm{Xv}^{\mathrm{e}}$ et le $\mathrm{XVIII}^{\mathrm{e}}$ siècle, entre Réforme et Contre-Réforme) que Foucault rencontre une dimension du rapport à soi, à son corps et à sa parole de vérité que les concepts d'individu discipliné et normalisé esquissés dans la première moitié de la même décennie, dans Surveiller et punir et encore sans doute dans La volonté de savoir ${ }^{5}$, ne permettaient pas de saisir entièrement. Dans les plis d'une généalogie de la sexualité qui remonte à la chair chrétienne, il se trouve face à un sujet ${ }^{6}$ complexe, irréductible à l'individu assujetti,

occidental et de la direction de conscience, telle qu'elle se développe à partir du concile de Trente ». Daniel Defert, "Chronologie », in M. Foucault, Euvres, t. 2, éd. Frédéric Gros, Paris, Gallimard, 2015, p. xxvir. Une première version du texte est achevée probablement au début de l'année 1978, mais Foucault commence à problématiser la question de la chair dès le milieu des années soixante-dix (voir le cours Les Anormaux, 1974-1975). Pour une reconstruction détaillée de l'histoire et de l'état du manuscrit, voir Philippe Chevallier, Michel Foucault et le christianisme, Lyon, ENS Éditions, 2011, en particulier p. $149-150$.

4. Michel Foucault, Le courage de la vérité. Le gouvernement de soi et des autres II. Cours au Collège de France. 1983-1984, éd. Frédéric Gros, Paris, Seuil/ Gallimard, 2009, p. 3.

5. Il est tout de même intéressant de souligner que c'est précisément à travers une généalogie religieuse de l'aveu de la vérité de soi, et en particulier de l'aveu de sa propre vérité à travers le sexe, que Foucault parle dans La volonté de savoir de "l'assujettissement des hommes; je veux dire leur constitution comme "sujets", aux deux sens du mot»; «c'est de ce jeu que s'est constitué, lentement depuis plusieurs siècles, un savoir du sujet; savoir, non pas tellement de sa forme, mais de ce qui le scinde ; de ce qui le détermine peut-être, mais surtout le fait d'échapper à lui-même »; M. Foucault, La volonté de savoir, in Euvres, " Pléiade », t. 2, op. cit., p. 659, 667.

6. On utilisera par la suite les termes de « sujet » et de « subjectivité » comme quasi-synonymes, comme le fait Foucault dans ses cours. Cela dit, la notion de « subjectivité » fait plutôt référence à l'ensemble de pratiques et d'expériences dans lesquelles le «sujet» se construit. Les deux termes ont évidemment chacun une longue histoire conceptuelle, qu'il n'est pas possible de traiter ici de manière analytique. Notre but est de montrer la complexité de la "généalogie » de ces notions dans le parcours foucaldien, en relation avec son exploration de l'histoire politique et religieuse des débuts de la modernité. 
irréductible à « l'âme prison du corps $»^{7}$, irréductible même à la «simple» prise de position du soi comme locuteur à l'intérieur d'un champ discursif.

Bien avant la réflexion sur la notion complexe de liberté politique mise en jeu par les libéralismes, dans le débat théologique autour de la chair, de sa liberté, de sa nature plus ou moins peccamineuse, plus ou moins capable d'obtenir le salut, Foucault met au jour la figure d'un sujet dont la construction implique constitutivement à la fois contraintes extérieures et action libre de soi sur soi. Dans les querelles théologico-morales autour de la Réforme, Foucault "découvre », pour ainsi dire, la subjectivité ${ }^{8}$ - une dimension bien différente pour Foucault de cette subjectivité psychologique, psychanalytique ou philosophique par rapport à laquelle il avait pris ses distances depuis les années cinquante et soixante: une subjectivité comme forme à construire du rapport à soi-même et aux autres (et à leurs injonctions de vérité), composition de forces hétérogènes, expérience conflictuelle et plurielle. L'étude du christianisme du début de l'ère moderne est un passage essentiel pour comprendre les développements ultérieurs de la démarche foucaldienne, et, en particulier, la nécessité de récupérer dans ses analyses une dimension subjective qui, pour n'avoir jamais été absente de ses préoccupations, n'était toutefois pas pensée dans la centralité de la relation à soi comme possibilité ouverte à la fois de conflit et de liberté.

La plongée dans la théologie morale chrétienne à l'époque des Réformes protestantes et catholiques permet d'ailleurs de comprendre aussi comment cette subjectivité est pour Foucault une expérience qui, depuis le début, est à la fois éthique et politique. À Luther ou à Cajetan, on peut attribuer beaucoup moins aisément qu'aux stoïciens et aux cyniques un «dandysme» de la pensée. Toutes ces figures incarnent et relancent mutuellement, tout au long des dix dernières années de vie de Foucault, des exigences théoriques et pratiques étroitement liées les unes aux autres, qui engagent en même temps la réflexion sur les formes de la

7. M. Foucault, Surveiller et punir, Paris, Gallimard, 1975 [« Tel», 1993], p. 38.

8. Il est significatif que le terme de subjectivité n'est présent dans aucun des deux ouvrages publiés par Foucault au milieu des années soixante-dix : Surveiller et punir et La volonté de savoir. 
subjectivité et sur les techniques de gouvernement des hommes. On connaît l'importance de la notion de pouvoir pastoral quant à l'émergence dans la pensée foucaldienne des analyses sur les rationalités et les pratiques gouvernementales, et il est évident que la notion de gouvernement comme "action sur l'action des autres » implique une prise en compte de la dimension subjective dans l'expérience politique. Il s'agit de parcourir «l'histoire des techniques de pouvoir, - disons plus exactement dans l'histoire de ce qui permet de constituer l'individu comme sujet $»^{9}$. Or, La chair et le corps démontre que, même avant l'étude des corpus éthico-politiques antique et patristique, c'est l'âge de la Réforme qui retient l'attention de Foucault et construit à ses yeux ce qu'on pourrait appeler une autre modernité du sujet: une subjectivité contre-rationaliste, contre-universelle, dont la conscience joue comme vecteur de conflit plutôt que comme instance de vérification absolue et transcendantale.

\section{« SUbJeCtiver » LA CHAIR}

Je commencerai par une exploration rapide de l'analyse foucaldienne de la théologie morale de la chair contenue dans le manuscrit inédit de La chair et le corps. J'essaierai ensuite de montrer en quoi cette lecture de la notion de chair est essentielle pour la généalogie du sujet et de ses rapports à la vérité que, à la fin de sa vie, Foucault affirme avoir voulue reconstruire tout au long de son parcours intellectuel.

Partons de La chair et le corps. Sous ce titre général, on trouve dans les archives Foucault des centaines de documents épars, ne composant pas un texte continu et révélant de la part de Foucault une reprise incessante et presque obsessionnelle de ses analyses (un même passage peut se trouver réécrit quatre, cinq, six fois avec des variations minimes de style et de contenu). Dans cet éparpillement inachevé, remontant sans doute, on l'a dit, au milieu des années soixante-dix, on peut tout de même retracer des lignes générales: Foucault se donne comme objet d'étude la question de la concupiscence et de la délectation dans les techniques de la

9. Bibliothèque nationale de France, archives Foucault, boîte 89, chemise 2. 
confession religieuse entre le $\mathrm{XV}^{\mathrm{e}}$ et le $\mathrm{XVIII}^{\mathrm{e}}$ siècle, à travers une généalogie de l'aveu, en particulier l'aveu des péchés de la chair, qui remonte au VIII ${ }^{\mathrm{e}}$ siècle de notre ère ${ }^{10}$. Ces études complexes de la confession de la luxure ont comme point de départ les changements produits par le développement des pratiques de direction de conscience sur les laïcs à partir du $\mathrm{Xv}^{\mathrm{e}}$ siècle (Foucault revient à plusieurs reprises sur l'analyse des ouvrages de saint Antonin de Florence, en particulier un manuel de confesseur daté de 1502 , le Confessorum Refugium ${ }^{11}$ ), et plus précisément les bouleversements dans les pratiques de dire-vrai sur soi apportés par la Réforme protestante et les ripostes de la Réforme catholique. Dans ce tournant bien spécifique de l'histoire moderne, Foucault dessine en particulier le passage d'une codification juridique et relationnelle des fautes portant sur les actes commis par les individus, à une grille d'exploration du péché qui descend au plus profond de l'intériorité du sujet avouant, qui recherche la vérité de sa faute dans les plis de ses désirs, dans l'élan de ses plaisirs, dans les mouvements de son corps et de sa conscience aux limites du volontaire et de l'involontaire.

Ce sont des idées que l'on trouvait déjà exposées, mais beaucoup plus synthétiquement, dans le cours au Collège de France Les anormaux (leçons du 19 et 26 février 1975). Avant le concile de Trente, le questionnement confessionnel des péchés sexuels portait sur les infractions aux règles qui codifiaient les actes selon leur nature et les personnes selon leur statut sociojuridique. Il existait une forme permise d'acte sexuel, celui qui avait lieu entre un homme et une femme unis par le mariage - avec quelques limitations supplétives : la femme ne doit pas être enceinte, la contraception est interdite, la finalité reproductive doit ordonner toute conjonction charnelle, etc. Les rapports sexuels

10. Sur la diffusion de l'obligation d'aveu dans l'espace catholique à partir du XIII ${ }^{\mathrm{e}}$ siècle, cf. Jean Delumeau, L'aveu et le pardon, Paris, Fayard, 1990 ; Librairie générale française, 1992, p. 9 : «Le vécu religieux quotidien s'en trouva bouleversé. Les prêtres et les laïcs se virent confrontés aux multiples difficultés de l'aveu, de l'évaluation des fautes et de l'appréciation du regret. Il fallait demander et obtenir le pardon. Mais à quelles conditions ? ». Ce travail reprend et prolonge des recherches élaborées par le Groupe de La Bussière : Pratiques de la confession: des Pères du désert à Vatican II, 15 études d'histoire, Paris, Éditions du Cerf, 1983.

11. Angelo Perilli (Saint Antonin), Confessorum refugium atque naufragium portus tutissimus “Defecerunt”, Lugduni, J. de Vingle, 1502. 
accomplis hors de ce cadre étaient considérés comme transgressifs, selon un catalogue différentiel des actes interdits (adultère, viol, inceste, sodomie, bestialité, etc.) en fonction de leurs modalités et des partenaires. Les nouvelles techniques de confession posttridentines vont s'appliquer plutôt au corps même du confessé ch $^{12}$ - ou mieux, à la «subjectivité » de son corps, que Foucault identifie à la « chair» : à savoir, « ses gestes, ses sens, ses plaisirs, ses pensées, ses désirs, l'intensité et la nature de ce qu'il éprouve lui-même $»^{13}$.

Dans l'un des dossiers manuscrits se rapportant à La chair et le corps, Foucault affirme qu'il y a un passage d'un ordre « juridicopénitentiel » à un ordre «correctif-directionnel $»^{14}$; et dans ce dernier, dans les formes directionnelles de la pénitence qui se diffusent et s'institutionnalisent à l'aube de la modernité, toute dimension de relation passe nécessairement par une injonction à la fois d'intériorisation et de naturalisation. D'une part, au centre de la confession du péché de luxure, on ne trouve plus les actes relationnels mais le corps et l'âme individuels, leurs impressions et leurs mouvements, le processus du désir conçu comme indéfini et suspendu dans le vide. D'autre part, ce mouvement d'intériorisation - ou pour mieux dire de subjectivation (on verra mieux plus loin dans quel sens j'utilise ce terme) - implique que le péché ne peut plus se concevoir comme une transgression de la loi qui est aussi et en même temps une sortie de la nature comme règle établie des actes. Le péché est un débordement de la nature dérivant des mécanismes de cette nature même : c'est le désir inattendu et irrépressible, le plaisir non maîtrisé qui excède les limites naturelles, les mouvements du corps brisant l'équilibre entre ce même corps et son guide rationnel. Pour le dire avec une formule paradoxale, le péché devient la «naturalité contre-nature »

12. «En défendant l'adultère, Dieu a défendu en même temps toute sorte d'impureté, capable de souiller le corps. De plus le libertinage intérieur du cœur est également défendu », Catéchisme du Concile de Trente (1566), chap. xxxiv ; voir aussi les chapitres XXI-XXIV sur le sacrement de pénitence (demandant d'une part une contrition sincère, préconisée pour tous les fidèles au moins une fois par an, à travers l'aveu «entier et complet» de ses péchés « mortels» au prêtre confesseur ; et d'autre part des pratiques de satisfaction de ces fautes).

13. M. Foucault, Les anormaux. Cours au Collège de France. 1974-1975, éd. Valerio Marchetti, Antonella Salomoni, Paris, Seuil/Gallimard, 1999, p. 173.

14. BnF, archives Foucault, boîte 64, chemise 9. 
de l'homme : il est à la fois le fruit des tentations qui inclinent tout naturellement l'homme au mal et un désordre fondamental venant briser l'ordre fixé par la nature. La nature elle-même se trouve alors mise en question par tout un débat qui en fait le modèle du bien moral et la possibilité toujours ouverte de transgression : on est précipité «dans l'infini d'un débat $[. .$.$] sur l'ordre sexuel où$ on expliquera que ceci ou cela est prohibé par le fait que c'est contraire à la nature, et où on justifiera qu'il y a des prohibitions par le fait qu'à la laisser faire, la nature est sans règle propre $»^{15}$.

On peut suivre ce même passage d'un ordre juridique à un ordre naturalisé et correctif-directionnel à travers une autre notion-clé pour la généalogie foucaldienne du discours sur la chair sexuelle : la concupiscence. Si, en effet, le concept de concupiscence est l'objet premier de la confession du péché de luxure, au cœur de son histoire, la manière de concevoir ce noyau d'expérience change radicalement aux yeux de Foucault au tournant des $\mathrm{XV}^{\mathrm{e}}$ et $\mathrm{XVI}^{\mathrm{e}}$ siècles. De principe explicatif et forme générale de la culpabilité (la forme mauvaise de la volonté qui détermine le péché), elle se transforme en un brouillage extrêmement complexe du volontaire et de l'involontaire qui se révèle dans des mouvements variés de volupté corporelle, négligence spirituelle, consentement implicite aux désirs inappropriés, incapacité de résistance aux pénétrations du Malin etc., jusqu'à l'acte peccamineux lui-même (l'émission de semence, notamment). La concupiscence devient un mot pour ce territoire trouble aux confins de la volonté, où la nature et ses limites, la rationalité et ses tentations, la lutte entre Dieu et le Diable se mêlent et déterminent un champ multiple de combat pour la volonté libre. Ce qui est en jeu ce n'est alors plus l'ensemble des lois et leurs transgressions ni le simple acte du corps ou de la volonté. C'est précisément la chair, au sens de la «subjectivité du corps » concupiscent, désirant et résistant, avec ses pulsions rebelles et ses désirs sournois, à l'emprise de la volonté ou de la conscience. «La chair, c'est la subjectivité même du corps, la chair chrétienne, c'est la sexualité prise à l'intérieur de cette subjectivité, de cet assujettissement de l'individu à lui-même ${ }^{16}$. L'homme

15. Ibid.

16. M. Foucault, « Sexualité et pouvoir », Dits et écrits II. 1976-1988, éd. Daniel Defert et François Ewald, Paris, Gallimard, 2001, texte n 233, p. 566. 
sexuel de la modernité se construit et se comprend comme un sujet de la concupiscence : un sujet incité à rechercher indéfiniment la vérité - sa propre vérité de sujet - dans les plis de sa chair, dans ses affects et ses mouvements involontaires, dans un combat incessant avec ce qui, en lui, se cache à lui-même. Cette dimension de la subjectivité comme expérience de soi fragmentée et toujours à recomposer est, me semble-t-il, la «découverte» fondamentale de Foucault dans la théologie morale tridentine. Ainsi peut-il écrire dans La chair et le corps :

Si le corps [dans le péché de luxure] échappe à l'âme, c'est dans la mesure où celle-ci se lie à elle-même dans un rapport qui prend la forme du plaisir pris au plaisir ; la chair que manifeste la matérialité du corps s'enracine dans une relation de soi à soi. Il s'agit donc ici d'une subjectivation de la chair elle-même, et non plus de l'acte par le biais de son imputation à un auteur. [C'est] la possibilité de concevoir la chair comme une forme fondamentale de subjectivité et de pouvoir saisir et analyser celle-ci à travers les mouvements de la chair ${ }^{17}$.

\section{Et encore :}

Ce qui s'y trouve mis en question, ce n'est pas un rapport, c'est un sujet. Ou plutôt une subjectivité, avec les flexions de ses automatismes et de ses consentements, avec les éveils et les limites de sa conscience, avec les formes, les mouvements et les occasions de ses plaisirs. [...] Le sexuel commence à s'inscrire non plus tellement dans une problématique de l'alliance avec ses codes, mais dans une problématique du sujet et de la vérité. Le sexuel va se mettre à fonctionner comme sexualité ${ }^{18}$.

\section{SUJETS RÉFORMÉS}

Dans ce procès de «subjectivation» du corps par la chair, un tournant fondamental est constitué par la diffusion des théories

17. BnF, archives Foucault, boîte 88 , chemise 7 ; nous soulignons. Il est important de rappeler qu'on a affaire ici à des fragments de chapitres-brouillons, auxquels Foucault n'a pas souhaité donner une forme achevée pour la publication. Il demeure néanmoins que des expressions comme «subjectivation de la chair » et « la chair comme une forme fondamentale de subjectivité » sont extrêmement significatives, si on les rapporte aux développements ultérieurs de la pensée de Foucault. Il faut se demander comment il utilise ici les termes «forme fondamentale» et «subjectivité / subjectivation». Qu'est-ce qu'une forme fondamentale de subjectivité pour Foucault, dans cette période de son travail? Qui, ou mieux, quelles techniques « donnent» cette forme ?

18. BnF, archives Foucault, boîte 64, chemise 9, nous soulignons. 
luthériennes et plus généralement protestantes de la chair et des péchés, ainsi que par les ripostes théologiques et politiques du côté catholique. On peut suivre cet « affrontement » entre les Réformes, capital pour Foucault, grâce à deux axes de réflexion et de débat (et on sait combien ce débat fut acharné et violent tout au long du $\mathrm{XVI}^{\mathrm{e}}$ siècle, et même au-delà) : 1) les positions respectives de la nature et du péché dans la doctrine de la luxure ; 2) la valeur de la confession et du dire-vrai sur soi-même pour le salut de l'âme, entre juridiction et justification.

Pour le premier point, Foucault fait jouer une opposition stratégique fondamentale entre Luther et Cajetan, qui correspond du point de vue conceptuel à un antagonisme entre la chair et la luxure. On a vu, en effet, combien la définition même du péché lié à la transgression du sixième commandement (« Tu ne commettras point d'adultère ») est pour Foucault une construction historique. Elle conditionne d'ailleurs la conception historique du sujet même qui commet cette transgression, et elle change entre les $\mathrm{XV}^{\mathrm{e}}$ et $\mathrm{XVII}^{\mathrm{e}}$ siècles, passant d'une vision juridique (infraction d'une loi) à une vision «physiologique» du péché (le mal s'insinuant de manière subtile et inquiétante dans le corps même du pécheur, dans ses désirs, dans ses plaisirs). D'où une difficulté de plus en plus accrue pour penser le péché en termes d'imputabilité d'une action à un sujet-individu libre. On se meut plutôt aux confins de la volonté, là où la liberté se brouille et se mêle à la possession intime du mal pénétrant le corps. Mais cette pénétration est toujours aussi un jeu inavouable de séduction qu'il faut, sinon vouloir, du moins accepter, et qui implique donc une dimension de capitulation et de volupté coupable. Les grandes affaires de possession à l'âge classique sont sur ce point tragiquement significatives : le diable est le grand Autre qui nous dépossède de notre subjectivité, de notre volonté libre, mais qui demande en même temps l'engagement de la responsabilité subjective qui l'appelle, l'accepte, et en jouit. Les « aveux de la chair ${ }^{19}$, dont Foucault veut faire la généalogie,

19. Dans le manuscrit de la préface écrite pour l'édition allemande de La volonté de savoir, («Sexualität und Wahrheit», in Der Wille zum Wissen, Frankfurt am Main, Suhrkamp Verlag, 1977), Foucault change le titre du deuxième tome de son Histoire de la sexualité de La chair et le corps en Les aveux de la chair (1. La volonté de savoir; 2. Les aveux de la chair; 3. La croisade des jeunes gens; 4. Population et races; 5. La femme, la mère et l'hystérique; 6. Les pervers). 
changent par conséquent de paradigme, passant d'une analyse des actes à une analyse des mouvements les plus obscurs et secrets de la chair comme « subjectivité du corps ». On se dirige vers l'âge de la «sexualité » comme expérience subjective et «psychologisée » de la vérité concernant la dimension sexuelle.

Faut-il pour cela affirmer que la nature humaine est en tant que telle peccamineuse, qu'elle n'est pas capable d'opposer au mal la force efficace de sa propre volonté rationnelle et libre ? Telle est, pour Foucault (qui simplifie évidemment un débat théologique fort complexe pour l'indexer à ses propres exigences théoriques), la position de Luther. La Réforme identifierait la concupiscence avec la chair et le péché de luxure : la concupiscence serait le signe de la chair comme nature déchue de l'homme, privée par le péché originel de la possibilité de se sauver par ses propres forces morales et donc indexée inévitablement - naturellement, pourrait-on dire sur la luxure, à savoir la racine de tous les péchés. La nature luxurieuse et la chair demeurent liées par l'expérience subjective de la faiblesse concupiscente comme noyau fondamental de la vie de l'homme sur terre, qu'il revient au seul don de la grâce de libérer ${ }^{20}$. Semper peccator, semper penitens, semper iustus ${ }^{21}$. «La théologie morale de Luther tend à identifier le péché et la chair et à faire de la concupiscence la forme subjective de leur unité $»^{22}$.

La réaction stratégique du «front » catholique passe, d'après Foucault, par une dissociation de la chair et de son péché, en élaborant une théorie de la luxure qui ne la rabat pas sur la chair elle-même. Cajetan joue un certain thomisme poussé à l'extrême contre Luther, contre un certain augustinisme de la Réforme. Ce

BnF, archives Foucault, boîte 51, chemise 3. Le texte de cette préface retraduit en français par Jacques Chavy («Sexualité et vérité ») a été repris dans Dits et écrits II. 1976-1988, op. cit., texte $\mathrm{n}^{\circ} 190$, p. 136-137.

20. «Moraliter: A carne adversaria spiritus quis liberabit? Nunquid ego vel homo ? Non, quia hinc video, quod errat qui seipsum vult salvare, et est homo mendax. Sed gratia dei per Ihesum Christum liberat triumphatque nos ab isto Pharaone », Dr. Martin Luthers Werke. Kritische Gesamtausgabe (Weimar/Köln, Böhlau, à partir de 1883), t. 4, p. 268, lignes 24-27. Sur la question de la liberté et de l'intériorité dans la doctrine de Luther, voir Philippe Büttgen, Luther et la philosophie, Paris, Vrin, 2011, en particulier p. 247-283 (je reprends les citations de Luther dans cet ouvrage).

21. Vorlesung über den Romerbrief (1515-1516), sur l'Épitre paulinienne aux Romains (Rm 7, 25), in Dr. Martin Luthers Werke. Kritische Gesamtausgabe, op. cit., t. 56, p. 347, lignes 2-6.

22. BnF, archives Foucault, boîte 88, chemise 4. 
n'est pas la nature humaine en tant que telle qui a été corrompue par le péché originel; ce dernier constitue pour chacun le risque toujours ouvert du péché, auquel pourtant l'expérience de la chair ne se réduit ni ne s'indexe nécessairement. Foucault reprend, on l'a dit, les théories de Cajetan, l'un des premiers théologiens catholiques qui se confronte au défi de la Réforme : les venerea comme ensemble des actes et plaisirs qui concernent la génération humaine sont à distinguer soigneusement de leurs excès et de leurs abus (superfluitas ${ }^{23}$ ). Il y a un «bon usage » de la chair, qui est finalisé à la procréation humaine et qui, comme tout acte bon - Aristote docet -, implique un plaisir propre et en aucune manière condamnable. Ce qui incline au péché, c'est l'usage impropre de ces venerea: les plaisirs mal placés, les désirs contre-nature, le désordre introduit dans la naturalité et la rationalité de l'existence humaine. "Consensus, delectatio, venerea; ce sont là les trois points essentiels d'une théorie de la luxure qui ne donne pas congé à une théorie de la chair, mais permet d'y faire passer un ordre des choses, des plaisirs, et de la volonté, et donc d'y opposer le désordre d'un péché imputable à un sujet $»^{24}$.

La citation l'affirme très clairement : cette théorie de la luxure où ni le corps ni sa chair comme expérience sexuelle subjective ne sont à condamner comme essentiellement peccamineux, n'a évidemment pas pour but premier de «sauver les plaisirs de la chair », mais de sauver la possibilité d'attribuer la responsabilité d'un acte, et partant de son péché, à un sujet, selon un paradigme qui veut se maintenir dans un cadre juridique et qui constitue pour Foucault le propre de la pastorale et des pratiques confessionnelles catholiques par rapport aux techniques de soi réformatrices - j'y reviendrai. L'enjeu est «de permettre de sauver une part ou un aspect de la nature, de donner prise à une définition précise d'actes fautifs, enfin de fixer le point de responsabilité, et donc les règles d'imputation de l'acte à un sujet $»^{25}$. Cajetan se fait porte-parole

23. Dans les notes en bas de page du manuscrit de La chair et le corps, Foucault se réfère au manuel en ordre alphabétique écrit par Cajetan en 1525 à l'intention des confesseurs (Summula peccatorum, article Luxuria), et à l'usage de superfluitas dans son commentaire de l'Épitre aux Éphésiens (cf. Ientacula Novi Testamenti, Romae, 1925).

24. BnF, archives Foucault, boîte 88, chemise 4.

25. Ibid. 
d'un thomisme naturalisant qui va peut-être trop loin par rapport aux orientations générales de la théologie morale catholique du $\mathrm{XVI}^{\mathrm{e}}$ siècle, il convient de le répéter, mais qui représente pourtant pour Foucault « la ligne de défense » du catholicisme : la luxure joue comme « une arme contre la conception protestante de la chair $»^{26}$.

Il est évident que ce qui intéresse le plus Foucault dans tout ce discours autour de la chair, du péché, de la luxure, ce sont les différentes formes de subjectivité qui se cristallisent autour des théories et des pratiques religieuses luthériennes et catholiques. Ou pour mieux dire, c'est le fait que la révolution incarnée par la Réforme, étudiée à travers l'histoire de la confession, et l'histoire de l'aveu, sexuel en particulier, impose de repenser radicalement la forme de rapport à soi et à la vérité impliquée par l'expérience sexuelle au début de l'âge moderne. Face à l'obligation de direvrai sur soi, dans un contexte de mise en question radicale de la possibilité pour l'individu d'atteindre le salut à travers cette même enquête pénitentielle, le sujet se trouve confronté à toute son épaisseur problématique. Qui suis-je ? Nature déchue ou « image» de Dieu ? Raison ou chair pécheresse ? Conscience libre ou sujet de l'institution ecclésiastique? Une nouvelle problématisation du soi s'impose : la modernité des conflits religieux est véritablement l'«âge de la subjectivité », le lieu de sa découverte pour les analyses foucaldiennes.

Le deuxième point de différence entre les doctrines protestantes et catholiques, souligné par Foucault est une démonstration ultérieure de cette centralité prise par la question du sujet, et passe à nouveau par la problématisation du caractère « juridique » de la confession. Il s'agit en effet de marquer la différence entre l'aveu catholique et ses reprises protestantes ${ }^{27}$, à travers trois axes qu'on peut décrire comme une double récusation et une reprise. Encore une fois, la reconstruction historique foucaldienne est schématique et orientée au filtre de son argumentation personnelle; elle passe rapidement sur beaucoup de points essentiels comme la distance

26. Ibid.

27. Sur l'histoire de la confession dans le premier protestantisme: Emil Fischer, Zur Geschichte der evangelischen Beichte, 2 vol., Leipzig, 1902-1903, réimp. Aalen, Scientia-Verlag, 1972, et Ilse Tobias, Die Beichte in den Flugschriften der frühen Reformationszeit, Frankfurt a. M., Peter Lang, 2002. 
entre les différentes confessions de la Réforme et les ambiguïtés de la pensée luthérienne elle-même. Cela n'implique pas pour autant qu'elle soit fausse ou non pertinente. Suivons-la donc, sous l'angle de la double récusation d'abord.

Porté par la vague d'indignation suscitée par la pratique des indulgences, Luther s'en prend, en effet, avant tout à la forme juridique et sacramentaire de la pénitence, selon sa forme élaborée par les institutions ecclésiastiques depuis le Moyen Âge, les XII ${ }^{\mathrm{e}}$ et XIII ${ }^{\mathrm{e}}$ siècles en particulier. La vente du pardon de Dieu par ses représentants sur terre en est l'expression la plus scandaleuse, mais elle est sous-tendue selon Luther par une conception de la grâce divine comme d'un tribunal à "pénitence tarifée »: Dieu concéderait le pardon selon un catalogue précis d'actes imputables à un sujet, de peines nécessaires pour expier tel ou tel péché et d'œuvres de charité pouvant contrebalancer une série de fautes. Or, Luther rappelle avec force que Dieu n'est pas un bureaucrate de tribunal, et que sa justice demeure inexplicable et inatteignable, une force active de grâce qui donne la foi et une miséricorde qu'aucune action humaine ne peut susciter ni justifier. "Il faut penser "justification" et non "juridiction". [...] Retour donc à une théologie rigoureuse du salut par-delà les élaborations juridiques qui, en oubliant que Dieu était Dieu, faisaient collaborer l'homme à son propre rachat $»^{28}$.

La pénitence ne sera alors pas un sacrement non plus, parce qu'aucune formule rituelle, aucun geste institué ne peut laver le péché du cœur de l'homme. Le ministre du culte ne détient plus sur terre les clés du royaume céleste; seul Dieu peut donner à chacun d'entre nous la force salvatrice de la foi. Et pourtant, cela n'implique pas l'absence dans le monde protestant de pratiques de confession, au sens d'une parole de vérité sur soi prononcée dans le cadre d'un rapport à un autre comme guide. Au contraire, le déplacement de ces pratiques hors du cadre sacramentel - incarné à l'âge de la Contre-Réforme par le dispositif très matériel du confessionnal dans les Églises -, ainsi qu'un rapport directement revendiqué de sa propre conscience avec la parole divine, provoque une démultiplication des actus veritatis et des techniques pénitentielles qui leur sont liés. Pourtant, c'est une

28. BnF, archives Foucault, boîte 88, chemise 3 . 
reprise qui déplace à la fois la forme et l'objet de cette véridiction de soi. Il ne s'agit plus de la récitation exhaustive de la liste de ses péchés selon un catalogue préconçu, ni de la réponse à une série de questions de la part du prêtre-ministre. La pénitence peut prendre plusieurs figures différentes, selon les Églises: « la forme d'une confession faite à Dieu dans le secret du cœur, d'un aveu fait publiquement devant la communauté réunie, de confidences faites à un frère, de conseils et d'incitations demandés à un pasteur; mais aussi de libres conversations à l'intérieur de la communauté, de récits d'expérience et de journaux intimes ${ }^{29}$. L'objet de ces explorations de soi change aussi. Luther considère comme trop lourdes et finalement inutiles l'enquête méticuleuse et l'injonction à la verbalisation indéfinie de "soi-même comme auteur de fautes passées », que la pastorale catholique avait progressivement institutionnalisées comme obligation pour tout chrétien, au moins une fois par an. Il préfère ramener la pénitence à «l'authentification de la foi actuelle et le déchiffrement des signes de la prédestination: plutôt que le catalogue judiciaire des imputations, le relevé des marques de Dieu à travers les événements d'une vie ». La vérité se fait découverte du secret de la foi. « Au lieu de renforcer le modèle judiciaire d'un aveu portant sur des actes et sollicitant le pardon d'un juge, - "voilà ce que j'ai fait, voilà ce qui est à absoudre" »-, dire la vérité sur soi-même « répand la forme d'une interrogation sur soi : "qui est donc celui que je suis et qui échappe à mon propre regard ? Élu ou réprouvé, aimé ou rejeté ? Jacob ou Ésaïe ?" » ${ }^{30}$.

\section{UNE CONTRE-HISTOIRE DE LA MODERNITÉ}

Tout ce long détour par la théologie morale du $\mathrm{XVI}^{\mathrm{e}}$ siècle montre clairement pourquoi la période de la Réforme et de la Contre-Réforme est considérée, selon une définition déjà évoquée et formulée par Foucault dans La chair et le corps, comme le grand « âge de la subjectivité ». La subjectivité émerge comme problème - «le problème du "sujet", comme élément dans un rapport

29. Ibid.

30. Ibid. 
d'autorité, comme pôle d'une relation de connaissance, et comme individu qui doit à la fois attendre et faire son salut $»^{31}$. Il ne s'agit pourtant pas de la part de Foucault d'une reprise détournée et rapide du topos de la modernité comme lieu de naissance de l'individu, de la "première personne » des révolutions religieuses, politiques, scientifiques. Les analyses foucaldiennes dessinent au contraire, en creux, une véritable « contre-histoire » du sujet de la modernité qui, plus qu'elle n'en dévoile la vérité intime, en problématise l'évidence première. C'est précisément une critique subtile des mythes modernes du Sujet. La subjectivité mise en jeu par les luttes religieuses autour de la réforme n'est pas le Sujet absolu de la philosophie et de la science, garant des fondements rationnels de la connaissance vraie. Il ne s'agit pas non plus de l'Individu comme atome de la pensée politique, ni de la Conscience revendiquée comme libre et autonome dans l'expérience de la foi $^{32}$. Foucault ouvre plutôt cet immense domaine d'analyse qu'est la subjectivité comme champ de lutte et réseau gouvernemental - l'entrelacement de rapports à soi, aux autres et à la vérité (à la fois imposée et librement revendiquée), qui sera au cœur de ses dernières analyses. La «découverte» foucaldienne du «pli » des techniques de soi, pour le dire avec Deleuze ${ }^{33}$, ne remonte pas à

31. BnF, archives Foucault, boîte 88, chemise 10.

32. Il est très intéressant pour cela de mettre en relation les usages de la notion de sujet dans les manuscrits se rapportant au projet de La chair et le corps, et les récurrences du même concept dans Surveiller et punir, où le sujet reste encore lié à la dimension de l'individualisation assujettissante. Dans un passage du manuscrit inédit d'une version primitive de Surveiller et punir, Foucault affirme : «Le rapport de pouvoir, la procédure de marquage, la technique d'individualisation précèdent donc l'individualité et la subjectivité qui en sont les effets. On pourrait dire d'une certaine façon que la position d'objet anticipe sur la position de sujet ; que c'est comme objet de pratiques individualisantes que le sujet se constitue. [...] Contre cette prise par l'individualisation objective, on comprend bien qu'il y ait eu historiquement deux batailles : l'une contre la position souveraine du sujet en général, l'autre contre les procédures d'objectivation du sujet: bataille de l'abstraction, bataille de l'aliénation. Mais menée seule la bataille de l'abstraction laisse subsister comme forme d'existence concrète les procédures de l'objectivation; et menée de son côté la bataille de l'aliénation fait valoir le sujet en général, l'être humain libre et individuel comme élément fondamental et premier. En fait c'est contre la prise individualisante, constitutive et du sujet en général et de l'objectivation, que la bataille doit être menée. S'arracher à la prise individualisante, telle qu'elle a été historiquement constituante ». BnF, archives Foucault, boîte 49 .

33. Cf. «Les plissements, ou le dedans de la pensée (subjectivation) », in Gilles Deleuze, Foucault, Paris, Éd. De Minuit, 1986 [2004], p. 101-130. 


\section{l'Antiquité. Elle se retrouve dans ce carrefour fondamental pour notre modernité qu'est l'époque des Réformes.}

On pourrait sans doute déchiffrer tout cet «âge » si complexe «des» Réformes [...] comme une multiplicité d'efforts, poussés parfois jusqu'au schisme et à la guerre ouverte, pour «subjectiver » le rapport à l'Au-delà, le rapport à la Vérité et le rapport à l'Autorité. Et pour avancer dans la schématisation on pourrait suggérer que les protestants, luthériens ou calvinistes, ont cherché la subjectivation de l'institution religieuse dans la primauté de la foi, dans le rapport immédiat à l'Écriture, et dans la règle du libre examen. La ContreRéforme catholique ne pouvait admettre aucune de ces trois formes de subjectivation sous peine de remettre en question la structure de l'Église elle-même. Ce qui ne veut pas dire qu'elle n'a pas aidé, à sa façon, à cette grande poussée de la subjectivation : mais elle a eu tendance à la canaliser dans la pastorale, où justement l'autorité ecclésiastique était affirmée : subjectivation de l'âme sous la forme de l'examen dirigé, élaboration continue d'une conscience de soi, médiation de soi à soi par un rapport d'obéissance au confesseur. En somme du côté de la Réforme protestante on trouverait plutôt la tendance à une subjectivation individualisante de l'expérience religieuse (qui incline à éluder dans la foi le rapport d'autorité); et du côté de la Réforme catholique, la subjectivation religieuse de l'expérience de soi (qui incline à introduire le principe de la direction dans le rapport à soi). Mais il ne faudrait pas donner trop de rigidité à ce schéma, car dans la plupart des Églises protestantes [...] la pratique de l'examen, de déchiffrement de soi par soi, était non seulement pratiquée, mais valorisée. Et du côté de la Contre-Réforme, il est arrivé que le cadre pastoral à l'intérieur duquel on tentait d'inscrire la subjectivation religieuse, se soit trouvé par là même menacé ou débordé : soit parce qu'elle ramenait insidieusement à des formes de rapports immédiats et hors autorité à Dieu, soit encore parce qu'elle développait, d'individu à individu, d'âme à âme, des formes de rapport qui tendaient à échapper à l'institution hiérarchique de l'Église ${ }^{34}$.

La publication des cours au Collège de France de 1978 et $1979^{35}$ nous avait déjà dévoilé un chapitre de cette « autre histoire » de la modernité que Foucault dessine à travers l'histoire du christianisme,

34. BnF, archives Foucault, boîte 88, chemise 10.

35. M. Foucault, Sécurité, territoire, population. Cours au Collège de France. 1977-1978, éd. Michel Senellart, Paris, Seuil/Gallimard, 2004 ; Naissance de la biopolitique. Cours au Collège de France. 1978-1979, éd. Michel Senellart, Paris, Seuil/Gallimard, 2004. Sur la nouveauté marquée par les cours de 1977-1979 dans le parcours foucaldien, voir Michel Senellart, "Situation du cours", in M. Foucault, Sécurité, territoire, population, op. cit., p. 381-411 ; Id., « La critique de la raison gouvernementale », in Guillaume le Blanc, Jean Terrel (éd.), Foucault au Collège de France. Un itinéraire, Pessac, Presses universitaires de Bordeaux, 2003, p. 131-147. 
grâce à l'élaboration en particulier de la notion de "pouvoir pastoral $»^{36}$. Foucault fait du christianisme, du monachisme ancien en particulier et de ses pratiques de direction de conscience, la «matrice juridique et politique des États modernes ${ }^{37}$ : l'émergence de la «raison d'État» aux $\mathrm{XV}^{\mathrm{e}}$ et $\mathrm{XVI}^{\mathrm{e}}$ siècles est étroitement liée à la diffusion et multiplication de rationalités et de pratiques spécifiques de gouvernement des hommes dans la sphère politique, religieuse, sociale. Les procès de constitution des États nationaux ne sont alors pas à relire dans le cadre des conflits entre pouvoir temporel et spirituel, à travers une reprise et un «transfert » d'éléments de la potestas religieuse dans la sphère politique. Ils seraient plutôt à replacer dans la longue histoire des techniques "gouvernementales", laquelle a ses racines dans la pensée orientale et hébraïque antique : le pouvoir du «pasteur» des hommes, à la fois individualisant et collectif, s'exerçant omnes et singulatim $^{38}$. Entre le pape et l'empereur, le «ministre $»^{39}$. «Avec le $\mathrm{XVI}^{\mathrm{e}}$ siècle on entre dans l'âge des conduites, dans l'âge des directions, dans l'âge des gouvernements $»^{40}$.

Foucault intervient à sa manière dans le long débat sur le «théologico-politique » et la notion de sécularisation ${ }^{41}$, en les faisant grimacer, en en construisant la généalogie comme instance critique. Le grand «monstre froid» de l'État trouve ses racines matérielles dans les formes de gouvernement des hommes dont l'histoire remonte aux techniques de direction religieuses, et

36. Voir en particulier Philippe Büttgen, «Théologie politique et pouvoir pastoral », Annales. Histoire, Sciences sociales, 5, 2007, p. 1129-1154. Cf. aussi Arnault Skornicki, La grande soif de l'État. Michel Foucault avec les sciences sociales, Paris, Les Prairies ordinaires, 2015.

37. Arnault Skornicki, La grande soif de l'État. Michel Foucault avec les sciences sociales, op. cit., p. 150. L'auteur précise un peu plus loin : «Foucault raconte une tout autre histoire de la modernité politique que Heidegger, par exemple, ou encore le marxisme : ce qui compte, ce n'est pas tant la découverte du logos grec, que la bergerie chrétienne; moins la bourgeoisie révolutionnaire, que les moines des $\mathrm{III}^{\mathrm{e}}$ et $\mathrm{IV}^{\mathrm{e}}$ siècles de l'Empire romain tardif. Foucault l'affirme sans ambages : la spécificité du pouvoir en Occident, on la doit à l'Église chrétienne » ; ibid., p. 158.

38. M. Foucault, "Omnes et singulatim: Towards a Criticism of Political Reason », Dits et écrits II. 1976-1988, op. cit., texte n 291, p. 953-980.

39. M. Foucault, Sécurité, territoire, population, op. cit., p. 195.

40. Ibid., p. 236.

41. Philippe Büttgen, art. cit., et Jean-Claude Monod, La querelle de la sécularisation. Théologies politiques et philosophies de l'histoire de Hegel à Blumenberg, Paris, Vrin, 2002. 
pourtant il ne se réduit aucunement à elles. Or l'histoire de la chair et de la concupiscence entre Réforme et Contre-réforme constitue l'autre volet de cette contre-histoire de la modernité, en retrouvant les origines du «sujet» du pouvoir étatique dans les stratégies de mise en discours de la vérité de soi au sein des pratiques pénitentielles chrétiennes. «D'un côté, constitution d'une souveraineté rationnelle de l'État; de l'autre, constitution d'une subjectivité véridique de l'individu $\gg^{42}$. Une étude des «herméneutiques » de soi se dessine, dont Foucault remontera le fil à partir du christianisme tridentin jusqu'aux Pères de l'Église, et de là aux penseurs antiques ${ }^{43}$. Entre gouvernement des hommes et dire-vrai sur soi-même, Foucault esquisse une réflexion critique sur son actualité politique qui le conduira jusqu'à l' ' épreuve du réel du politique » platonicienne, jusqu'aux exercices spirituels stoïciens, jusqu'aux scandaleux corps-théâtres de vérité des cyniques.

Comment peut-on décrire le «grimacement» opéré par la généalogie foucaldienne à propos de l'histoire du sujet moderne? On pourrait dire, pour schématiser, que la subjectivité découverte et fondée par la modernitét ${ }^{4}$ assume trois figures fondamentales. Elle est : 1. un sujet à la fois de connaissance et de reconnaissance (valeur épistémique de la subjectivité ; l'Ego cartésien se découvre être le seul garant de l'évidence du vrai dans un procès qui est en même temps une découverte de sa propre vérité d'être pensant); 2. un sujet qui est gouverné et qui se gouverne lui-même par sa volonté propre, libre et rationnelle (valeur éthico-politique de la subjectivité ; l'Individu peut décider de déléguer ses propres droits à l'autorité souveraine, fût-ce une fois pour toutes ; le fondement du lien social comme contrat de volontés singulières et autonomes); 3. un sujet qui s'engage dans un procès de mise à jour de sa propre vérité naturelle (valeur anthropologico-psychologique de la subjectivité ; l'Homme naturel à la racine des sciences humaines,

42. BnF, archives Foucault, boîte 88, chemise 10 .

43. Cf. Michel Senellart, «Michel Foucault: une autre histoire du christianisme? », Bulletin du centre d'études médiévales d'Auxerre / BUCEMA [En ligne], Hors-série $\mathrm{n}^{\circ} 7,2013$, mis en ligne le 29 mars 2013, consulté le 10 septembre 2016. URL : http://cem.revues.org/12872 ; DOI : 10.4000/cem.12872.

44. Pour un survol bien construit de la question et une première bibliographie : Jean Baudrillard, Alain Brunn, Jacinto Lageira, «Modernité », Encyclopaedia Universalis, consulté le 10 septembre 2016, disponible en ligne (https://wwwuniversalis-edu-com.acces-distant.sciences-po.fr/encyclopedie/modernite/). 
dont la sociologie, la médecine, la psychanalyse essaieront tour à tour de dire l'essence véritable et le destin promis).

À partir de ces valeurs de la subjectivité, Foucault construit une généalogie à travers l'histoire chrétienne de la chair qui est une véritable parodie au sens nietzschéen du terme : le repérage de visages historiques différenciés du sujet qui peuvent inquiéter notre vision acquise, les conventions et habitudes de la pensée trop facilement acceptées. L'histoire généalogique est une critique qui montre «combien nous sommes loin de ce qui nous fonde, même si ce qui nous fonde est au plus près de nous $»^{45}$. Le sujet moderne n'est alors pas le fondement absolu et transcendantal de la connaissance vraie; il est le lieu où doit perpétuellement se faire et se refaire la recherche si instable d'une vérité de soi toujours engagée et jamais achevée. Il n'est pas l'individualité autonome qui fonde le vivre en commun; il est le produit historique et contingent d'une pluralité d'instances politiques et de rapports de force qui s'exercent sur lui et à partir de lui. Il n'est pas une nature que l'on peut décrire scientifiquement une fois pour toutes ; il est cette expérience menaçante où l'individualité et la naturalité se dissolvent, le risque ouvert de la pénétration dans soi de l'autre que soi, de la présence du Démon comme signe à la fois de la faiblesse naturelle de l'homme et de sa déchéance. Le cœur secret de la modernité n'est pas la lumière de la res cogitans cartésienne : c'est le retentissement violent et sanglant des conflits de religion, de la bataille pour le salut religieux et politique. Pudenda origo que non seulement les mythologies de la Raison et de l'Histoire dans les Lumières mais aussi un certain laïcisme contemporain (et même parfois foucaldien) voudraient peut-être faire oublier.

Dans la généalogie de la sexualité remontant à la conception chrétienne de la chair, Foucault découvre une dimension du sujet irréductible aux simples procès d'assujettissement, lesquels restent bien entendu présents dans les techniques pastorales. Il s'agit avant tout d'un sujet qui est contraint de découvrir en soi la profondeur d'un abîme qu'il ne maîtrise et ne maîtrisera jamais complètement, et qui pourtant définit son identité propre : une subjectivité toujours exposée au risque de pénétration de l'autre, une subjectivité éparpillée en fragments de voluptés et de délectations interdites et

45. BnF, archives Foucault, boîte 48, chemise 2 . 
contradictoires, un soi qui doit perpétuellement s'avouer dans sa propre vérité pour conjurer - et jamais définitivement, du moins dans cette vie terrestre - le risque de perdre son identité et sa consistance subjective. Foucault découvre, me semble-t-il, une subjectivité à plusieurs dimensions, qui se définit structurellement dans un réseau complexe de rapports de pouvoir exercés sur lui, mais ne peut pas se penser sans les multiples techniques de véridiction et de subjectivation que le sujet s'impose à lui-même et par lui-même, dans un effort éthique constant. On comprend ainsi mieux, à la fin de notre parcours, pourquoi j'ai pu affirmer que les racines chrétiennes de la généalogie foucaldienne de la subjectivité jouent déjà comme une critique ante litteram de toute interprétation « dandyste » et antipolitique du dernier Foucault. Pour fasciné que Foucault puisse être par des possibilités inédites de subjectivation « libre », par des rapports de soi à soi se construisant hors du cadre de la loi et de la norme comme ceux qu'il croit retrouver dans l'Antiquité, la subjectivité est avant tout pour lui une dimension sans laquelle les mécanismes de pouvoir spécifiques à la culture occidentale - comme ceux qui obligent les individus à dire le vrai sur eux-mêmes - ne seraient pas pleinement intelligibles.

En conclusion, Foucault touche, aux limites de la modernité, à une subjectivité comme expérience d'un sujet dissocié, pluriel, relationnel et agonistique. Dans notre époque de profonde crise de la rationalité moderne et d'inquiétants «retours » du religieux sur la scène politique, il faudrait récupérer la force heuristique de cette analyse foucaldienne d'un sujet qui n'est pas absent, effacé par la postmodernité «liquide » ou réduit à une simple fonction dérivée du discours ; un sujet qui n'est pas simplement dominé, dépendant de pouvoirs qui s'exercent sur lui de l'extérieur ; un sujet qui n'est pas absolument libre et fort de son rapport à la vérité, mais une dimension ouverte au cœur d'une lutte. Autrement dit, un sujet « qui découvre à lui-même et à l'autre cette part de soi qui lui échappe $»^{46}$.

arianna.sforzini@sciencespo.fr

46. BnF, archives Foucault, boîte 88, chemise 11. 